\title{
Primeros Estudios sobre los RAP derivados de Mezclas con Asfaltos Modificados (RMAP), en la elaboración de nuevas mezclas en caliente
}

\section{First Studies about RAP of a Hot Mix Asphalt with Modified Bitumen (RMAP), in the new hot mixes asphalt production}

Presentación: 6-7/10/2020

\section{Doctorando:}

\section{Nidia Nilda Estefanía Fretes}

Centro de Investigaciones Viales LEMaC-CIC-PBA, Facultad Regional La Plata y LEME, Facultad Regional Avellaneda, Universidad Tecnológica Nacional - Argentina nfretes@fra.utn.edu.ar

\section{Director/a:}

\section{Hugo Daniel Bianchetto}

\section{Co-director/a:}

\section{Héctor Luis Delbono}

\begin{abstract}
Resumen
El sistema de transporte de caminos juega un rol importante en el crecimiento y desarrollo de los países. La mayoría de las rutas nacionales están confeccionadas con pavimentos flexibles. El método convencional de fabricación de mezclas asfálticas en caliente utiliza dos recursos no renovables, como son los agregados pétreos y el asfalto. Este estudio analiza el uso del reciclado en caliente de una mezcla asfáltica, que reduce el empleo de materiales nuevos, alentando de esta manera al desarrollo de la denominada economía "circular". En particular, este RAP es derivado de una mezcla asfáltica con asfalto modificado con polímero y se le da aquí el nombre de "RMAP" ("M" por modificado). En el laboratorio del Centro de Investigaciones Viales LEMaC-CIC-PBA, se cuenta con tres tipologías diferentes de RMAP. En esta ocasión, se exponen los resultados preliminares derivados de la caracterización de los materiales componentes para la confección de una mezcla densa en caliente CAC D 19 con un 25\% de RMAP1 en peso, utilizando como ligante asfáltico virgen un CA-30. Dicho RMAP hasta su periodo de fresado cuenta con un periodo en servicio de 10 años aproximadamente. Para la obtención del asfalto envejecido se utilizó un Rotovapor. El porcentaje de asfalto envejecido en peso de RMAP se constató con el ensayo de calcinación. Con el asfalto modificado envejecido se prosiguió a ejecutar los siguientes ensayos: penetración, punto de ablandamiento, viscosidad rotacional con el viscosímetro Brookfield y el retorno elástico torsional en particular, para evaluar la presencia de propiedades elásticas según su origen. Se exponen los resultados que demuestran que, a pesar de estar envejecido el cemento asfáltico, aún conserva mayores prestaciones en comparación con un RAP derivado de una mezcla asfáltica con asfalto convencional, además de la notable reducción de asfalto y de agregado virgen en la producción de nuevas mesclas en caliente.
\end{abstract}

Palabras clave: Reciclado, mezclas en caliente, RMAP 


\begin{abstract}
The road transport system plays an important role to growth and development of the countries. Most of the national roads are made with flexible pavements. The conventional method of manufacturing hot asphalt mixes uses two non-renewable resources such as stone aggregates and asphalt. This study analyse the use of hot recycling of an asphalt mix, which reduces the use of the new materials, thus encouraging the development of the so-called "circular" economy. In particular, this RAP is derived from an asphalt mix with polymer modified asphalt and is called "RMAP" (modified, "M"). In the Road Research Center's labratory LEMaC-CIC-PBA there are three different typs of RMAP. On this occasion, the preliminary results derived from the characterization of the component materials for the preparation of a dense hot mix CAC D19 with 25\% RMAP1 by weight are presented, using a CA-30 as a virgin asphalt binder. This RMAP until its milling period has a service period of approximately ten years. To obtain the aged asphalt a Rotovapor was used. The percentage of aged asphalt by weight of RMAP was also obtained, which was verified with the calcination test. With the aged modified asphalt, the following tests were carried out: penetration, softening point, rotational viscosity with the Brookfield viscometer and the torsional elastic return in particular, to evaluate the presence of elastic properties according to their origin. The results are presented that show, despite being aged, the asphalt cement still contains higher benefits compared to a RAP derived from conventional mix asphalt, in addition to the notable reduction of asphalt and virgin aggregate in the production of new hot mixes.
\end{abstract}

Keywords: Recycling, hot mixes, RMAP

\title{
Introducción
}

Los caminos que componen la red vial propician el transporte y la circulación, tanto de productos como de personas, alentando al continuo crecimiento de un país. En nuestro caso particular, con la extensión a nivel territorial que presenta nuestro país, genera que el mantenimiento y la ampliación de la red vial tome una notable importancia. La aparición de las tecnologías de los asfaltos modificados propició mejoras en las características de los asfaltos convencionales frente a las nuevas condiciones a los que se ven expuestos, desde la amplitud del gradiente térmico hasta mayores solicitaciones por el aumento de los niveles de tránsito.(Rondón Quintana et al., 2008). En la Argentina se emplean estas técnicas hace más de dos décadas, debido a lo cual, hoy en día, las capas superficiales de mezclas bituminosas elaboradas con ligantes modificados con polímeros están culminando su periodo de usufructo debiendo ser fresadas, generando un material novedoso.

Las técnicas de reciclado se han ido incrementando a lo largo de los años. En el caso del uso de RAP en altas tasas su empleo conlleva a la reducción de $\mathrm{CO}_{2}$, en especial por la distancia de transporte en tramos cortos o inclusive nulos que genera el reciclado. En los trabajos relacionados a la rehabilitación de los caminos, está implícito que para su correcta ejecución se deberá fresar de manera parcial o total las capas envejecidas. En estos casos, los organismos involucrados en la gestión deberían contar con técnicas adecuadas para el uso y manejo del RAP, de lo contrario los costos de las rehabilitaciones de los pavimentos asfálticos se incrementarían de manera significativa para solventar los costos que implica la disposición de los materiales no reciclados (Leiva-Villacorta \& Vargas-Nordcbeck, 2017). En la última edición actualizada 2017 del PETG de la Dirección Nacional de Vialidad, para mezcla densas con aporte de RAP, se hace mención a la implementación de este material tanto para la ejecución de carpetas de rodamiento como para capas de base, tal como se puede apreciar en la Tabla 1, donde se muestran porcentajes de hasta un 50\% de RAP en peso de mezcla, según los niveles de tránsito a los cuales se vean expuestos. 


\begin{tabular}{|c|c|c|c|c|}
\hline \multicolumn{5}{|c|}{ Porcentaje en peso de agregado proveniente del RAP respecto del total del agregado } \\
\hline \multirow{2}{*}{ Tipo de capa } & \multicolumn{4}{|c|}{ Clasificación por Tránsito } \\
\cline { 2 - 5 } & $\mathrm{T} 1$ & $\mathrm{~T} 2$ & $\mathrm{~T} 3$ & $\mathrm{~T} 4$ \\
\hline Rodamiento & $\leq 30 \%$ & $\leq 40 \%$ & $\leq 50 \%$ & $\leq 50 \%$ \\
\hline Base & $\leq 40 \%$ & $\leq 50 \%$ & $\leq 50 \%$ & $\leq 50 \%$ \\
\hline
\end{tabular}

Tabla 1: Porcentajes de RAP en función a la tipología de la capa según PETG para mezclas densas en caliente con aporte de RAP de DNVedición 2017

En el caso particular de un RAP derivado de un asfalto modificado RMAP ("M" por modificado) ocurren dos fenómenos en conjunto: por un lado, el deterioro propio sufrido por el asfalto que conforma el medio dispersante y por el otro, la degradación del polímero, que es la fase dispersada, obteniéndose como resultado aquel que genere mayor incidencia (Larsen et al., 2012). Los componentes que contienen los asfaltos son: saturados, asfáltenos, resinas y aromáticos, los denominados SARA, mientras que, a los últimos dos se los puede agrupar como máltenos. Entonces, podemos concluir que los ligantes asfálticos están compuestos por: asfáltenos y máltenos. Debido al envejecimiento a corto plazo que sufren las mezclas asfálticas desde su elaboración hasta su puesta en obra, al estar expuestas a temperaturas en el orden de $\operatorname{los} 130^{\circ} \mathrm{C}$ a $160^{\circ} \mathrm{C} \mathrm{y,}$ el envejecimiento a largo plazo, generado por su condición en servicio ante su exposición al oxígeno, las altas temperaturas ambiente y los rayos UV, que propician la pérdida de los máltenos y el aumento de los asfáltenos, moléculas de mayor peso molecular que rigidizan al ligante asfáltico. Los procesos antes mencionados conllevan, en muchos casos, al empleo de rejuvenecedores para restituir internamente el funcionamiento de los asfaltos.

Partiendo de la premisa que los asfaltos modificados denominados "Premium" presentan mejores prestaciones que los asfaltos convencionales, en las mezclas densas en caliente a diseñar se emplearán como asfalto virgen un asfalto CA-30, al cual se le adiciona el asfalto de aporte de los diferentes RMAP, esperando de esta manera, igualar o incluso mejorar las características originales que el ligante asfáltico convencional posee.

\section{Desarrollo}

En el Centro de Investigaciones Viales LEMaC-CIC-PBA se cuenta con material proveniente de diferentes tipologías de mezclas asfálticas con asfalto modificado, tiempo en servicio y, un dato no menor, el equipo con el cual se desarrolló el fresado, dado que a futuro se estima analizar la incidencia de dicha acción mecánica generada por las picas, cantidad y distribución según la maquinaria disponible, lo cual seguramente genera alteraciones en el producto final. A continuación, en la Tabla 2, se muestran las tres tipologías de RMAP con las cuales se cuenta en el laboratorio.

\begin{tabular}{|c|c|c|c|}
\hline $\begin{array}{c}\text { RAP derivado de } \\
\text { mezclas con asfalto } \\
\text { modificado }\end{array}$ & Ubicación del tramo & $\begin{array}{c}\text { Tipología de } \\
\text { mezclas asfáltica } \\
\text { modificada de } \\
\text { origen }\end{array}$ & $\begin{array}{c}\text { Tiempo en años de } \\
\text { servicio }\end{array}$ \\
\hline RMAP1 & AU Ezeiza- Cañuelas, Buenos \\
Aires & SMA 19 & 10 a 12 \\
\hline RMAP2 & $\begin{array}{c}\text { RP 91 Cañada de Gómez-Totoras, } \\
\text { Santa Fe }\end{array}$ & CACD con AM3 & 1,5 \\
\hline RMAP3 & $\begin{array}{c}\text { Av 7 entre calle 54 y 57, La Plata, } \\
\text { Buenos Aires }\end{array}$ & CACD con AM3 & 20 \\
\hline
\end{tabular}

Tabla 2: Tipologías de RMAP con que se cuenta en el laboratorio del Centro de Investigaciones Viales LEMaC-CIC-PBA 
El ligante modificado envejecido se extrajo utilizando la técnica de destilación controlada en el Rotovapor, con el uso de un solvente (el diclorometano) que no necesita altas temperaturas para generar el "lavado" del RMAP (Bianchetto et al., 2006) Con el asfalto recuperado se realizaron ensayos empíricos de consistencia tales como: penetración, punto de ablandamiento y, debido a su origen, se determinó el retorno elástico torsional para evaluar su aptitud elástica pese a su estado de oxidación. En la Tabla 3 se exponen los resultados de dichos ensayos y en la Tabla 4, se establece la clasificación de los asfaltos modificados con polímeros para uso vial según la norma IRAM 6596. Comparando ambas Tablas, se puede observar que un asfalto modificado AM3, luego de estar en servicio ante solicitaciones importantes por ser el caso de una autopista durante aproximadamente 10 años, aun puede ser clasificado como un asfalto modificado AM1, conservando en menor proporción sus propiedades elásticas.

\begin{tabular}{|l|c|c|}
\hline Parámetros & $\begin{array}{c}\text { Valores } \\
\text { obtenidos }\end{array}$ & $\begin{array}{c}\text { Método de } \\
\text { ensayo }\end{array}$ \\
\hline Penetración $\left(25^{\circ} \mathrm{C}, 100 \mathrm{~g}, 5 \mathrm{~s}\right)(1 \mathrm{~d} . \mathrm{mm})$ & 20 & IRAM 6576 \\
\hline Punto de Ablandamiento $\left({ }^{\circ} \mathrm{C}\right)$ & 63 & IRAM 6841 \\
\hline Recuperación elástica por torsión $\left(25^{\circ} \mathrm{C}\right)(\%)$ & 12 & IRAM 6830 \\
\hline Viscosidad Brookfield a $100^{\circ} \mathrm{C}($ Poise $)$ & 148 & IRAM 6837 \\
\hline Viscosidad Brookfield a $135^{\circ} \mathrm{C}($ Poise) & 11,7 & IRAM 6837 \\
\hline
\end{tabular}

Tabla 3: Resultados de los ensayos del asfalto modificado envejecido recuperado del RMAP1

\begin{tabular}{|l|c|c|c|c|}
\hline Parámetro & AM1 & AM2 & AM3 & $\begin{array}{c}\text { Método de } \\
\text { ensayo }\end{array}$ \\
\hline Penetración $\left(25^{\circ} \mathrm{C}, 100 \mathrm{~g}, 5 \mathrm{~s}\right)(1 \mathrm{~d} \cdot \mathrm{mm})$ & $>20$ & $>50$ & $>50$ & IRAM 6576 \\
\hline Punto de Ablandamiento $\left({ }^{\circ} \mathrm{C}\right)$ & $>60$ & $>60$ & $>65$ & IRAM 6841 \\
\hline Recuperación elástica por torsión $\left(25^{\circ} \mathrm{C}\right)(\%)$ & $>10$ & $>40$ & $>70$ & IRAM 6830 \\
\hline
\end{tabular}

Tabla 4: Requisitos particulares de los asfaltos modificados según norma IRAM 6596

Con el agregado "lavado" del RMAP se prosiguió a desarrollar la granulometría, necesaria para la dosificación de la mezcla asfáltica densa en caliente, con $25 \%$ de RMAP en peso, que se propone diseñar. Situación similar, a la que estarán expuesto los RMAP dentro del tambor de la planta de producción, donde las elevadas temperaturas y el calor que le proporcionan los agregados pétreos vírgenes precalentados al mezclarse, producirán una disgregación del material. En la Figura 1 se muestra el RMAP en condición seca luego de estar en estufa a $60^{\circ} \mathrm{C}$ y después del "lavado" con el solvente. Mientras que, en la Figura 2 se expone la granulometría del RMAP1 en comparación con los husos establecidos para una SMA 19, donde se aprecia como la mezcla se ve afectada por la acción del fresado producido por las picas que contienen los tambores de la fresadora, además de la velocidad de circulación de la misma, parámetros que modifican en mayor o menor medida al producto final. Para poder resaltar esta condición, dentro de la gráfica granulométrica se marcó la posición de la abertura correspondiente al tamiz $\mathrm{N}^{\circ} 4$, el cual separa la distribución de los agregados grueso y los finos, dejando en evidencia el mayor aporte de agregados finos que provee el RMAP1 a la nueva mezcla. 


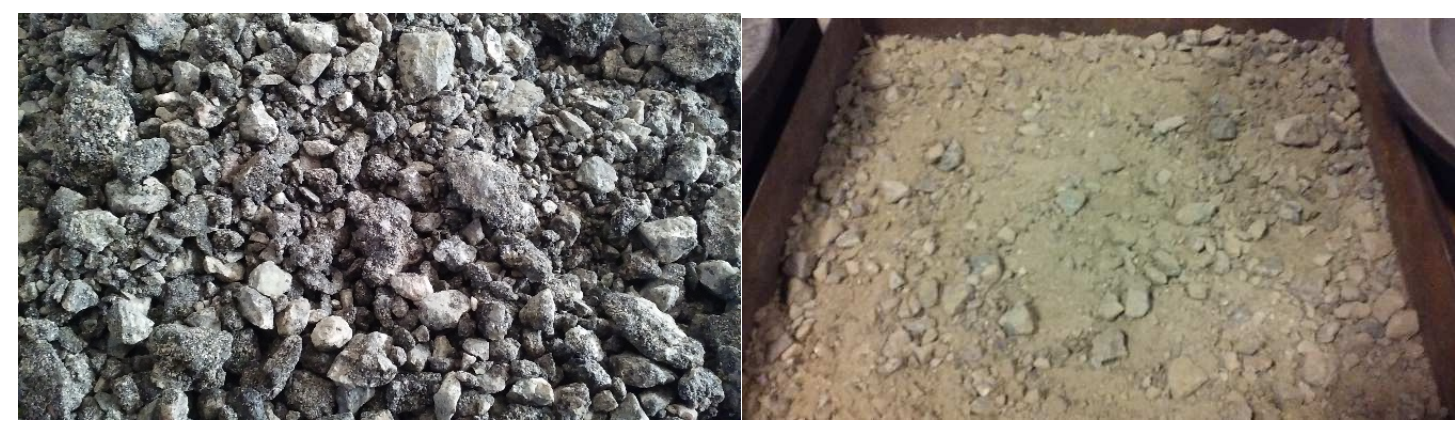

Figura 1: RMAP en la condición de secado en estufa a $60^{\circ} \mathrm{C}$ (Izquierda) RMAP luego del "lavado" (derecha)

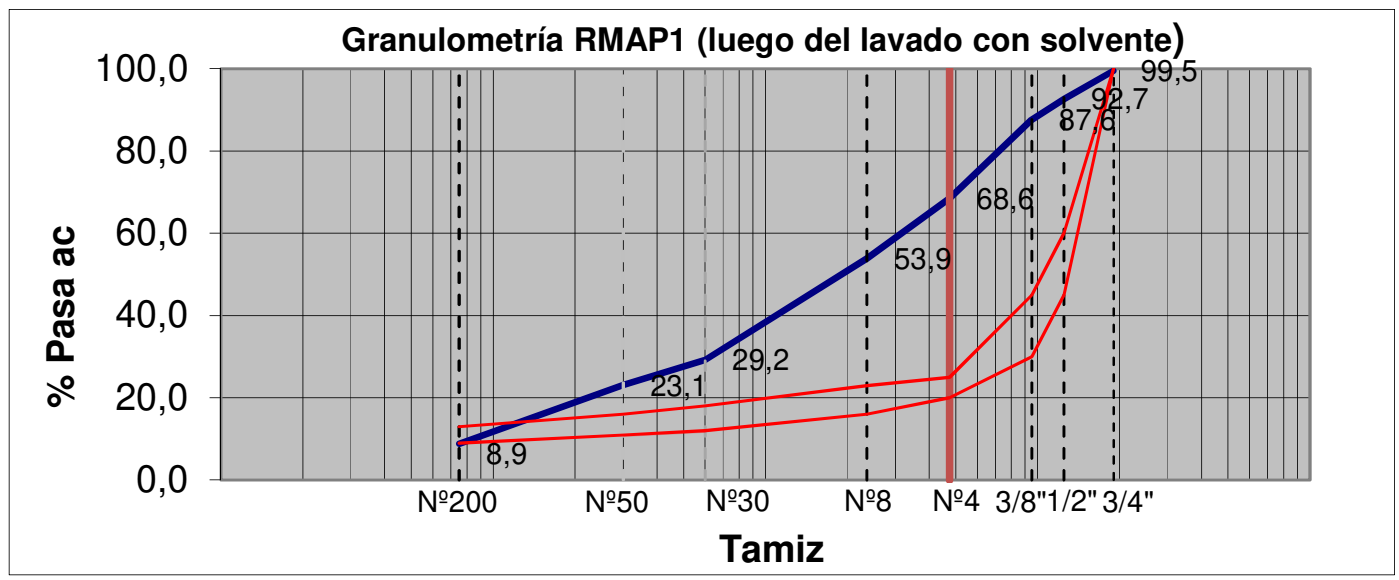

Figura 2: Granulometría del RMAP luego del "lavado" con solvente.

La caracterización de los demás componentes de la mezcla asfáltica, tales como los agregados pétreos vírgenes provenientes del sistema serrano de Tandilia, con graduación 0-6 y 6-20; el ligante asfáltico virgen CA-30 y el filler calcáreo, en este caso se utilizará cal hidráulica hidratada de origen comercial cumplen con los parámetros establecidos para uso vial con las normativas vigente según lo establecido en el PETG para mezclas densas en caliente y semicaliente con aporte de RAP.

Para el diseño de la mezcla se utiliza el Método Marshall. Los primeros ensayos volumétricos establecidos por el método han arrojado porcentajes óptimos de asfalto virgen adicionado menores a un $4 \%$ en peso de mezclas, valor menor que el habitual obtenido en el diseño de mezclas asfálticas densas. Para constatar el porcentaje de asfalto envejecido que aporta el RMAP a la nueva mezcla, se le realizó el ensayo de calcinación, el cual arrojo como resultado un 4,59\% en peso de RMAP. Todo lo antes mencionado constata los amplios beneficios que genera el uso del reciclado, al reducir el empleo de dos materiales no renovables y de elevado costo, como es el caso del cemento asfáltico y el agregado pétreo, además de la merma en los niveles de $\mathrm{CO}_{2}$. debido a la reducción de los trasladados al convertirse la propia vía en una cantera, contribuyendo a la disminución del impacto ambiental que estas actividades generan.

\section{Conclusiones}

Se debe resaltar el importante beneficio que genera la aplicación del reciclado, tanto desde el punto de vista medioambiental como económico. Los resultados preliminares expuestos muestran que el RMAP contiene propiedades notables en comparación con un RAP convencional, dato no menor para generar un uso óptimo de dicho material, ya que de lo contrario se estaría subestimando el potencial real que éste, aunque ha sufrido un deterioro en servicio, puede ofrecer a la nueva mezcla. Se demuestra así que, aunque el camino se encuentre deteriorado, sus componentes aún presentan 
características aprovechables, en particular el RMAP, que por su origen es un material de alta performance. En este caso se muestra, cómo un AM3 envejecido en servicio puede todavía clasificarse como un asfalto modificado, aunque de menor calidad, lo que genera mayor incentivo para continuar con el análisis y desarrollar una distinción desde la manipulación según el origen del RAP, para de esta manera aprovechar todas las propiedades que dicho producto novedoso puede proveer a las nuevas mezclas que con ellos se elabore.

En la continuidad de la investigación, se buscará profundizar el análisis reológico de cada uno de los asfaltos modificados envejecidos obtenidos de los RMAP con los cuales se cuenta, mediante el empleo de un reómetro de corte (DSR). Además, se efectuará un estudio específico integral de análisis macromolecular por espectrometría de dispersión de electrones(EDAX), Termo-gravimetría (TGA), calorimetría diferencial de barrido (DSC) y Espectroscopia infrarroja por transformada de Fourier(FTIR). Con todo el análisis mencionado se evaluarán las propiedades de este asfalto residual en comparación con el un asfalto convencional y un asfalto AM3 virgen, haciendo la salvedad que seguramente no tendrá las mismas características que el asfalto modificado utilizado para la confección de la mezcla asfáltica modificada, sobre todo respecto al RMAP3, que estuvo en servicio durante 20 años.

Para el caso de las mezclas asfálticas, una vez concluidos los diseños de mezclas mediante el método Marshall, se evaluará su grado de desempeño ante la fisuración por fatiga, la fisuración térmica (dato importante debido a la variación térmica que presentan la región sur y norte de nuestro país) y deformaciones permanentes o ahuellamiento (rutting).

Debido a la situación actual por la que estamos atravesando a causa de la pandemia, los estudios en el laboratorio han quedado detenidos momentáneamente. Se continua con la búsqueda bibliográfica y en la organización de las actividades para, apenas se pueda retomar la actividad, continuar con la investigación.

\section{Referencias}

Bianchetto, H. D., Miró Recasens, J. R., \& Pérez Jiménez, F. E. (2006). Resistencia al envejecimiento de las mezclas bituminosas en caliente: Beneficios y limitaciones de la incorporación de fílleres comerciales. Segunda parte: Estudios sobre los ligantes mediante ensayos reologicos y técnicas de analíticas macromolecular. Carreteras, 148, 20.

Larsen, D. O., Williams, E., Balige, M., Berardo, A., \& Gulo, L. (2012). Estudio de asfalto modificado envejecido en servicio para futuros proyectos de reciclados. Memorias del XVI Congreso Argentino de Vialidad y Tránsito, 12.

Leiva-Villacorta, I. F., \& Vargas-Nordcbeck, I. A. (2017). Mejores prácticas para diseñar mezclas asfálticas con pavimento asfáltico recuperado (RAP). 19, 10.

Rondón Quintana, H. A., Reyes Lizcano, F. A., Figueroa Infante, A. S., Rodriguez Rincón, E., Real Triana, C. M., \& Montealegre Elizalde, T. A. (2008, febrero). Estado del conocimiento del estudio sobre mezclas asfálticas modificadas en Colombia. Infraestructura Vial, 19. 